\title{
Deep Learning Based Air-Writing Recognition with the Choice of Proper Interpolation Technique
}

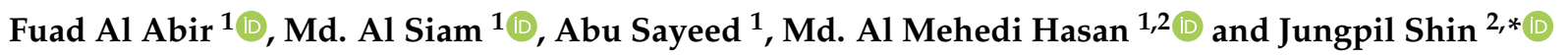 \\ 1 Department of Computer Science \& Engineering, Rajshahi University of Engineering \& Technology, \\ Rajshahi 6204, Bangladesh; 1603021@student.ruet.ac.bd (F.A.A.); 1603008@student.ruet.ac.bd (M.A.S.); \\ abusayeed@cse.ruet.ac.bd (A.S.); mehedi@u-aizu.ac.jp (M.A.M.H.) \\ 2 School of Computer Science and Engineering, The University of Aizu, Aizuwakamatsu 965-8580, Japan \\ * Correspondence: jpshin@u-aizu.ac.jp
}

check for updates

Citation: Abir, F.A.; Siam, M.A.; Sayeed, A.; Hasan, M.A.M.; Shin, J. Deep Learning Based Air-Writing Recognition with the Choice of Proper Interpolation Technique. Sensors 2021, 21, 8407. https:// doi.org/10.3390/s21248407

Academic Editor: Stefano Berretti

Received: 1 December 2021

Accepted: 13 December 2021

Published: 16 December 2021

Publisher's Note: MDPI stays neutral with regard to jurisdictional claims in published maps and institutional affiliations.

Copyright: (c) 2021 by the authors. Licensee MDPI, Basel, Switzerland. This article is an open access article distributed under the terms and conditions of the Creative Commons Attribution (CC BY) license (https:/ / creativecommons.org/licenses/by/ $4.0 /)$.

\begin{abstract}
The act of writing letters or words in free space with body movements is known as airwriting. Air-writing recognition is a special case of gesture recognition in which gestures correspond to characters and digits written in the air. Air-writing, unlike general gestures, does not require the memorization of predefined special gesture patterns. Rather, it is sensitive to the subject and language of interest. Traditional air-writing requires an extra device containing sensor(s), while the wide adoption of smart-bands eliminates the requirement of the extra device. Therefore, air-writing recognition systems are becoming more flexible day by day. However, the variability of signal duration is a key problem in developing an air-writing recognition model. Inconsistent signal duration is obvious due to the nature of the writing and data-recording process. To make the signals consistent in length, researchers attempted various strategies including padding and truncating, but these procedures result in significant data loss. Interpolation is a statistical technique that can be employed for time-series signals to ensure minimum data loss. In this paper, we extensively investigated different interpolation techniques on seven publicly available air-writing datasets and developed a method to recognize air-written characters using a $2 \mathrm{D}-\mathrm{CNN}$ model. In both user-dependent and user-independent principles, our method outperformed all the state-of-the-art methods by a clear margin for all datasets.
\end{abstract}

Keywords: air-writing recognition; interpolation; time-series data; human-computer interaction; convolutional neural network

\section{Introduction}

In the last decade, we have grown accustomed to interacting with the digital world in various ways. Touchscreens and other electronic devices are common means for people to connect to the internet. The use of smartphones and other physical devices imposes the additional burden of transporting them and taking them out of one's pocket to interact with them. The primary goal of next-generation technologies is to eliminate the necessity for intermediary physical devices, for instance, smartphones [1]. Virtual and augmented reality appears to be leading the way for the next generation of such technology, with output being projected directly into the eyes of the user(s) via specialized glasses [2]. Speech recognition is a well-studied approach that is thought to be a natural and intuitive way of interacting with technologies. However, speech recognition does not fulfill all parameters needed to communicate with technologies [1]. Gesture recognition is a method that has received a lot of interest in recent years which can be a communication method for next-generation technologies [2]. New technologies based on cameras, acceleration sensors, photosensors, and electromagnetic and auditory signals are emerging as new mediums of interaction employing gestures as an alternative to standard keyboards, touchpads, or other pushing and touching instruments [3]. In particular, for writing methods, traditional writing in touch sensors does not fit into Virtual Reality (VR), Augmented Reality (AR), and gesture-based 
technologies. To meet the requirement of touchless writing methods of next-generation technologies, air-writing is a promising solution. Air-writing is known as the act of writing letters or words with fingers or hand movements in free space [4]. It can be regarded as a special case of gesture, while the gesture is performing any kind of predefined movement in the air.

However, the recognition of air-writing characters is not a simple task [4]. Characters are distinguished from generic gestures by their fine-grained movement and they can be written in various ways by different people. In conventional writing, alphabets and numbers are written in a multi-stroke way in pen-and-paper-like systems [5]. Air-writing is different from the conventional writing system. Movements caused by lifting a pen are significantly less noticeable while writing in the air. Since users do not have the sense of touching anything, such as a pen or a piece of paper, they cannot see what is being written as they could see while writing on a piece of paper. As a result, the users may lose their sense of writing orientation in the space [2]. Despite all these difficulties, the recent advancements in the domain are promising.

In recent years, smartphones with in-built sensors have become widely available. Sensor data can be collected and preprocessed as per user requirement by creating a mobile application for the data collection process [2]. Hence, the domain of sensor datasets generated by smartphones is becoming larger day by day. Generally, while collecting the sensor data for gesture recognition, a user makes a gesture while holding or wearing motion sensors such as a gyroscope and/or an accelerometer $[2,5,6]$. On the other hand, the main challenge of building an air-writing recognition model using sensor data is the variability of signal length. Due to the nature of writing and data-recording procedures, variable signal length is evident [2], whereas deep learning methods such as the convolutional neural network require fixed-length signals in the training and prediction process. Fixed-length padding and truncation is a widely used process that pads or discards values from the start and/or end of a signal [7]. It is a simple method to implement but results in huge data loss, and therefore, the model is unable to capture significant features. On the contrary, interpolation is a statistical technique that predicts unknown values based on known values [8]. It ensures the fixed length of the signals by mapping the signal data into a predefined fixed length as a whole without discarding any portion of the signal. Therefore, data loss is minimal. Multiple interpolation techniques are available and well studied in the domain of image processing $[9,10]$. We investigated those various interpolation techniques on time-series data to obtain the fixed-length signals, i.e., employed interpolation methods on one-dimensional data instead of two-dimensional data. Furthermore, a well-structured, fine-tuned deep learning model can ease the training process and yield better accuracy in prediction. We designed a 2D-CNN model following the best practices proved in the literature and obtained state-of-the-art accuracy on seven publicly available datasets.

The organization of the rest of this paper is presented as follows: Section 2 presents the related works. Section 3 describes the dataset and the recognition methodology. In Section 4, the experimental analysis and evaluation of the methods are reported. Section 5 summarizes the paper emphasizing our contributions.

\section{Related Work}

Several researchers have recommended using motion sensors for gesture detection in recent years [6,11]. Liu et al. used an accelerometer signal which was captured from a Wii remote and recognized a predefined set of eight gestures using a DTW-based method [6]. The DTW-based method is a well-studied approach to deal with time-series data and air-writing recognition [12-14]. Chen et al. determined air-writing to be better than virtual keyboards in typing accuracy [4,15]. The authors also looked into identifying the beginning and end of each letter by the segments of the writing signal in a continuous data stream. A Wii remote was also used by $\mathrm{Xu}$ and $\mathrm{Xue}$, where the users were given instructions about the order of movement for each of the air-written letters [16]. Li et al. used mobile-phone-captured motion signals performed by users and an LSTM-based 
deep neural network architecture to differentiate between twelve different handwritten characters consisting of six uppercase letters and six digits [17].

Since users perform air-writing by hand, the signals received from palm-worn devices may lead it to be harder to recognize the activities [2]. However, several studies have depicted that it is possible to classify the gestures from palm-worn devices $[18,19]$. Amma et al. recognized air-written letters with high accuracy using motion sensors positioned on the palm [20]. Xu et al. recognized textual input from wrist sensor data obtaining an accuracy of $98 \%$ [21]. Lin et al. investigated the orientations of the surfaces in which users were to write characters, the stabilization (support) point of the hand, and the rotation injection technique for data augmentation which uses a rotation matrix [22]. They obtained a remarkably high accuracy of $99.99 \%$ to recognize 62 characters by 10 subjects with a machine-learning-based approach. Chen et al. investigated real-time fingertip detection in frames captured from smart glasses. They built a synthetic dataset using Unity3D and proposed a modified mask regional convolutional neural network. Their method could detect fingertip for air-writing in a minimal length of time for each frame [23]. Kim et al. experimented with the WiTA dataset, which contains air-writing data for Korean and English alphabets collected by RGB cameras [24]. Bastas et al. experimented with handwritten digits, ranging from 0 to 9 , which were structured as a multidimensional time-series data obtained via a Leap Motion Controller (LMC) sensor [25]. Tsai et al. suggested a reverse time-ordered algorithm to efficiently filter out unnecessary lifting strokes while writing in the air. To overcome the problem of different writing styles of different users, a tiered arrangement structure was presented by sampling the air-writing results with varied sample rates [26]. Arsalan et al. suggested an air-writing system based on a network of sparse radars and a 1D DCNN-LSTM-1D transposed DCNN architecture that can rebuild and identify the drawn character [27].

Moazen et al. attempted to recognize air-writing with a dataset containing 100 sets of samples of all 26 English letters collected from a single subject [28]. Uysal et al. proposed RF-Wri, a device-free machine-learning-based air-writing recognition framework that can differentiate 26 capital letters [29]. Yanay et al. allowed the users to write with their hands in the air naturally while capturing the motion signals by smart-bands [2]. In this experiment, the accelerometer and gyroscope signals were collected from the smart-bands to create a dataset of 15 sets of English alphabet for 55 subjects each. Finally, an average accuracy of $83.20 \%$ with the user-independent method and $89.20 \%$ with the user-dependent method was obtained in their experiment. To extract air-writing trajectories captured by a single web camera, Hsieh et al. proposed a hand-tracking algorithm [30]. Alam et al. experimented with a trajectory-based air-writing system where a depth camera was used which could track the fingertip to collect three-dimensional (3D) trajectories. They collected 21,000 trajectories and developed LSTM, CNN, and nearest-neighbor-based approaches and managed to obtain $99.17 \%$ accuracy [5]. Alam et al. proposed a trajectory-based air-writing character recognition system called CNN-LSTM, which used a combination of convolutional neural networks (CNNs) and long short-term memory (LSTM) to achieve 99.63 percent and 98.74 percent accuracy in the RTD and RTC datasets, respectively [31]. Alam et al. developed a technique for a finger-joint tracking-based character recognition system that uses 3D information to monitor the finger-joint and use the distance between the thumb tip and another finger-joint to identify a numerical digit, alphabet, character, special key, or symbol. Firstly, a single-hand-based digit recognition system was presented, which could be utilized with either the left or right hand. Secondly, a two-handed writing method was demonstrated, in which both hands were engaged at the same time. They achieved an overall accuracy of $91.95 \%$ for single-hand recognition and $91.85 \%$ for double-hand recognition, respectively [32]. In the absence of paired inertial and trajectory data, $\mathrm{Xu}$ et al. suggested an Air-Writing Translator model for learning bi-directional translation between trajectory and inertial domains. The researchers tested the suggested model on two publicly available datasets, 6DMG (in-air handwriting dataset) and CT 
(handwritten trajectory dataset), and showed that the model can reliably translate between the inertial and trajectory domains [33].

Our study differs from that of the existing studies in multiple aspects: (1) Though interpolation techniques are well studied in the digital image domain, they are often overlooked for time-series data [34]. In this paper, we experimented with various interpolation techniques on different publicly available air-writing datasets $[2,5,35,36]$ and yielded the best interpolation technique for air-writing time-series data. (2) Proposing a well-structured, tuned deep learning model is necessary to gain the most of the data. We proposed a 2D-CNN model following the best practices proved in the literature [37-39]. These measures result in state-of-the-art performance, outperforming all the existing methods in both user-dependent and user-independent training principles by a clear margin for each of the seven air-writing datasets.

\section{Materials and Methods}

\subsection{Dataset Description}

We used a total of seven publicly available air-writing datasets in this research $[2,5,35,36]$. All of the datasets are different in the number of classes, the number of users or subjects (interchangeably used throughout the paper), the number of features, and the data acquisition method. Among the datasets, all of them were experimented with a user-dependent training principle and five of them were experimented with a user-independent training principle. In the user-dependent principle, samples from all users were considered for training and cross validation was employed for testing purposes. This is also known as the user-mixed principle [33]. Meanwhile, the user-independent principle overcame the necessity of the user registration process prior to testing. Here, one user was held off from testing while the rest of the users' data were used for training. In Table 1, we present the summary of the datasets used in this study.

Table 1. Summary of the datasets used in this study.

\begin{tabular}{|c|c|c|c|c|c|c|}
\hline \multirow{2}{*}{ Dataset } & \multirow{2}{*}{ No. of Classes, $n$} & \multirow{2}{*}{ No. of Features, $n$} & \multirow{2}{*}{ No. of Users } & \multirow{2}{*}{ No. of Samples } & \multicolumn{2}{|c|}{ Training Principle } \\
\hline & & & & & User Dependent & User Independent \\
\hline $\mathrm{RTD}^{*}[5]$ & 10 & 2 & 10 & 20,000 & $\checkmark$ & $x$ \\
\hline $\mathrm{RTC}^{*}[36]$ & 26 & 3 & 10 & 30,000 & $\checkmark$ & $x$ \\
\hline Smart-band [2] & 26 & 6 & 55 & 21,450 & $\checkmark$ & $\checkmark$ \\
\hline 6DMG-digit [35] & 10 & 13 & 6 & 600 & $\checkmark$ & $\checkmark$ \\
\hline 6DMG-lower [35] & 26 & 13 & 6 & 1470 & $\checkmark$ & $\checkmark$ \\
\hline 6DMG-upper [35] & 26 & 13 & 25 & 6500 & $\checkmark$ & $\checkmark$ \\
\hline 6DMG-all [35] & 62 & 13 & 25 & 8570 & $\checkmark$ & $\checkmark$ \\
\hline
\end{tabular}

* The authors did not disclose user data. Therefore, training in user-independent principle cannot be performed.

\subsubsection{Smart-Band Dataset}

The smart-band dataset created by Yanay et al. contains air-writing data collected from 55 subjects [2]. Each subject wrote 15 sets of all 26 letters in the English alphabet in the air wearing a smart-band on their wrist. So, the dataset contains 390 samples of air-written letters per subject and there are 21,450 samples in total in the dataset.

The subjects wore the 'Microsoft Band 2' smart-band. The smart-band motion sensors, e.g., accelerometer and gyroscope, were used to record motion measurements in each sample. Both the accelerometer and gyroscope data were taken from three different axes $(X$, $\mathrm{Y}$, and $\mathrm{Z}$ ) with a maximum sampling rate of $62 \mathrm{~Hz}$. To collect the signals from the motion sensors, an android mobile application was developed which ran on a Samsung Galaxy S8 smartphone. The smart-band was worn in the wrist of the hand with which the subject normally wrote, and the smartphone was held with another hand. The smartphone was connected to the smart-band via Bluetooth to collect data. Among the 55 subjects, 28 were females and the rest were males, while 46 subjects were right-handed, and the remaining 9 subjects were left-handed. 


\subsubsection{Six-Dimensional Motion Gesture (6DMG) Datasets}

The 6DMG dataset is a collection of alphanumeric air-writing characters, numerics, and gestures [35]. It was gathered using a hybrid framework in which an inertial sensor recorded tri-axial acceleration and tri-axial angular velocity, and an optical tracking device recorded the spatial coordinate trajectory. As a result, the 6DMG dataset includes both inertial and trajectory domain information. There is a total of 62 characters in this dataset, with 26 uppercase letters, 26 lowercase letters, and 10 numerals. The uppercase letters data were collected from 25 subjects, whereas only 6 subjects were used to compile data for the digit and lowercase dataset. Overall, the dataset comprises a total of 8570 samples, with 600 numeric samples, 6500 uppercase letter samples, and 1470 lowercase letter samples.

\subsubsection{RealSense-Based 3D Trajectory Digit and Character (RTD-RTC) Datasets}

RealSense-based 3D Trajectory Digit and Character Datasets, abbreviated as RTD and RTC, respectively, are publicly available air-writing datasets containing the sequence of trajectory captured while writing English alphabets or digits in the free space. Both of the datasets were collected by Alam et al. [5,36]. In the RTC dataset, users wrote the English alphabets in front of sensor devices. The sensor data were gathered as a trajectory sequence. The fingertip was considered a substitute for a pen in the conventional pen-paper writing method. Users could write a character in front of an Intel RealSense SR300 camera, and the camera recognized the fingertip and collected it as a trajectory sequence. The direction of writing was a little different from the conventional multistroke style of writing, and it was written in a unistroke style [36]. In the RTD dataset, users wrote digits in front of the same device settings as RTC. The digits were written by the users in a defined manner [5].

Both the RTD and RTC datasets contain data as a sequence of trajectory, where each tuple indicates a trajectory. In each of the tuples, there are values of X-coordinate, Y-coordinate and Z-coordinate in the 3D Cartesian coordinate system, which was taken from the sensor device. However, in our experiment, we found that considering all three of the coordinate values from the RTD dataset produced meaningless output. Rather, only considering the $X$ and $Y$ coordinates made sensible outputs for the dataset. Therefore, the number of features for RTD and RTC dataset is 2 and 3, respectively, (see Table 1).

\subsection{Data Preprocessing}

In this work, we experimented with various interpolation methods while maintaining a fixed signal length of the air-writing sensor data. As the dataset is well balanced across the letters and free from missing values, no further data processing measures were necessary.

\subsubsection{Optimal Signal Length Selection}

To feed the data into deep learning architectures such as the convolutional neural network for training and prediction, maintenance of a fixed length is a must. Due to the nature of the data acquisition procedure, the length of the signals is likely to differ. As we used interpolation techniques described in the previous section (see Section 3.2.2), we had to find a suitable length to shape all the signals. We applied two following techniques to obtain this goal:

1. We could consider the mean of the signal lengths such that the fixed length nearly split the data in half. Half of the signal length was less than the fixed length, so we had to upsample the data to increase the length. We downsampled the other half of the signals where the length of the signals was greater than the fixed length.

2. As loss occurs in downsampling the data, we could consider upsampling the maximum number of signals so that the data loss was kept to a minimum and the signal length was manageable.

\subsubsection{Fixed-Length Signals Using Interpolation Techniques}

Interpolation is a statistical technique to predict probable unknown values based on known values [8]. It is widely used in image processing for reshaping images without the 
loss of the quality of the image or the visual experience and for improving the quality of the image. We used these interpolation techniques to obtain fixed-length signals to feed into the deep learning model without aggressive data loss.

In recent years, image processing methods with interpolation have gained importance for the capability of improving bad resolution images preserving the characteristics of the image [9]. The quality of the processed image is dependent on the chosen interpolation algorithm. There are different types of interpolation algorithms that have previously been developed. Among those algorithms, nearest neighbor, Bilinear, Bicubic, and Lanczos interpolation methods are widely used in different fields [10]. In Figure 1, we show the effects of different interpolation methods for upsampling and downsampling time-series signals. The interpolation techniques used in this paper are described below.

A Bicubic Interpolation: The Bicubic interpolation is the advanced version of cubic interpolation in a two-dimensional regular grid. The interpolation surface obtained here was smooth. Polynomial, cubic, or cubic convolution algorithm was used here. The cubic convolution determines the gray level value using the 16 closest pixels to the specified input coordinates and assigns the value to the output coordinates. The Bicubic interpolation kernel, $W(x)$ [40] is defined as follows,

$$
W(x)= \begin{cases}(a+2)|x|^{3}-(a+3) x^{2}+1 & \text { for }|x| \leq 1 \\ a|x|^{3}-5 a|x|^{2}+8 a|x|-4 a & \text { for } 1<|x|<2 \\ 0 & \text { otherwise }\end{cases}
$$

where $a$ is generally -0.50 or -0.75 .

B Lanczos Interpolation: To smoothly interpolate the value of a digital signal between samples, the Lanczos filter is employed. Here, each sample of the given signal was mapped to a translated and scaled copy of the Lanczos kernel, $L(x)$. The Lanczos kernel is a normalized sinc function which is windowed by a sinc window. The sinc window used is defined as the central lobe of a horizontally stretched sinc function $\operatorname{sinc}(x / a)$ for $-a \leq x \leq a$.

$$
L(x)= \begin{cases}\operatorname{sinc}(x) \operatorname{sinc}\left(\frac{x}{a}\right) & \text { if }-a<x<a \\ 0 & \text { otherwise }\end{cases}
$$

Equivalently,

$$
L(x)= \begin{cases}1 & \text { if } x=0 \\ \frac{a \sin (\pi x) \sin \left(\frac{\pi x}{a}\right)}{\pi^{2} x^{2}} & \text { if }-a \leq x<a \text { and } x \neq 0 \\ 0 & \text { otherwise }\end{cases}
$$

where $a$ is a positive integer determining the size of the kernel, generally 2 or 3 . The Lanczos kernel contains $2 a-1$ lobes. Among them, the number of positive lobes at the center is $a$ and the other $a-1$ lobes are situated at each side which are alternating negative and positive lobes. For a one-dimensional signal with samples $s_{i}$, the value interpolated at an arbitrary real argument $x, S(x)$ is obtained by the discrete convolution of those samples with the Lanczos kernel,

$$
\sum_{i=\lfloor x\rfloor-a+1}^{\lfloor x\rfloor+a} s_{i} L(x-i)
$$

where the filter size parameter is defined as $a$. The sum is bounded in such a way that the kernel is 0 outside of the boundary [41].

C Bilinear Interpolation: The use of linear polynomials to generate new data points within the range of a discrete set of known data points is known as linear interpolation. 
Bilinear interpolation is accomplished by first performing linear interpolation in one direction and then repeating the process from the opposite direction. In bilinear interpolation, a value for a random position is determined by the weighted average of the four closest values. In the sense of image processing, the four closest values can be regarded as the four closest coordinates to the specified coordinate for which the value is to be determined. In this method, two linear interpolations are performed. One linear interpolation is performed in a direction and the next is performed in the perpendicular direction. The output is smoother than the original input value set. When all distances between the data points are equal, then the interpolated value is their sum divided by four. Here, the interpolation kernel, $H(x)$ is

$$
H(x)= \begin{cases}0 & \text { if }|x|>1 \\ 1-|x| & \text { if }|x|<1\end{cases}
$$

where $x$ is the distance between two points to be interpolated.

D Nearest Neighbor Interpolation: Nearest neighbor interpolation is the most simple interpolation technique [42,43]. In this method, each interpolated output value is generated with the closest sample point in the input. This method produces discontinuous interpolated data [44]. The interpolated point $X_{i}$ is determined by

$$
X_{i}= \begin{cases}X_{B} & \text { if } i<\frac{a+b}{2} \\ X_{A} & \text { if } i \geq \frac{a+b}{2}\end{cases}
$$

where $a$ and $b$ are the indexes of $x_{A}$ and $x_{B}$ and $a<i<b$.

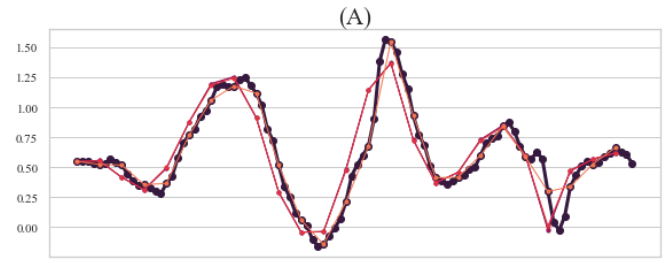

(C)

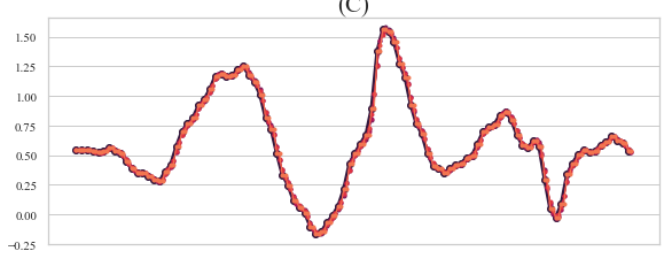

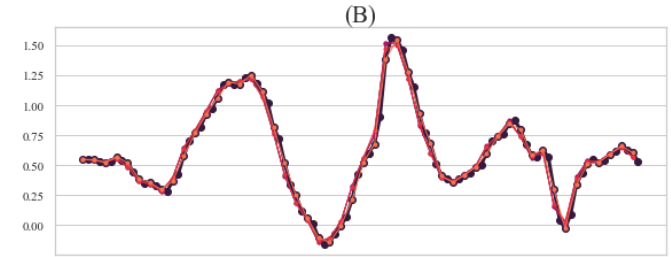

(D)

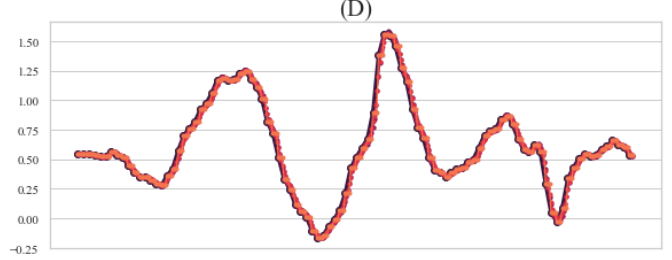

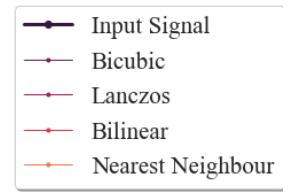

Figure 1. Different interpolation techniques applied on time-series data for upsampling and downsampling. (A) for 1/4 downsampling (signal length from 100 to 25), (B) for 1/2 downsampling (signal length from 100 to 50), (C) for $2 \times$ upsampling (signal length from 100 to 200), (D) for $4 \times$ upsampling (signal length from 100 to 400 ). The input signal was taken from the smart-band dataset.

\subsection{Convolutional Neural Network Architecture}

A convolutional neural network, abbreviated as CNN, is a type of deep neural network for processing raw visual data, inspired by the organization of the visual cortex of animals [45,46] and made to learn spatial hierarchies of features, from low-level to highlevel patterns, automatically and adaptively using convolutional and pooling layers and activation functions. CNNs are widely employed in computer vision tasks. Classification, object localization and detection, segmentation, and pose estimation are some of these tasks, to name a few. Lately, they have gained popularity in the research area of human activity recognition [47-49]. They have also been used for the classification of time-series data obtained from accelerometers, gyroscopes, and other sensors [50-53]. We proposed a convolutional neural network following the best practices that adapts well for air-writing recognition utilizing time-series data from various sensors. 
Our proposed convolutional neural network architecture was composed of four groups of layers other than the input layer, where the first three groups consisted of a couple of two-dimensional convolution, maxpooling, and dropout layers for feature extraction. We flattened the output from the third convolutional group, and a dense layer accompanying dropout was employed with the softmax activation function to gain the prediction. Except for the prediction layer, we used Rectified Linear Units (ReLUs) as the activation function throughout the network.

The input of the network was the tensor of format: $l \times f \times 1$, where $l$ is the signal length, $f$ is the number of features (time-series signals) in the dataset. This tensor was therefore propagated through the convolutional layers. Each of the convolutional groups was constructed using conv-conv-maxpool-dropout layers, sequentially. The core objective for consecutive convolutional layers without pooling layers is to replace a single layer with a larger receptive field rather than skipping any pooling. It is a widely used construct for developing convolutional neural network [37,54-56]. We incorporated two non-linear convolutional layers instead of a single one with a larger filter size to make the decision function more discriminative. Additionally, this approach decreased the number of trainable parameters [37].

Dropout has been an integral part of deep neural networks since their inception [57]. $\mathrm{Wu}$ and $\mathrm{Gu}$ studied the effects of dropout on different layers of CNNs and showed that the dropout of maxpooling and fully-connected layers performed best [38]. Therefore, we used dropout after every maxpooling layer and in the fully-connected layer where the percentage, $p$ values of the dropouts were chosen according to the suggestions given by Park and Kwak [39]. The network architecture specification is provided in Table 2, considering the smart-band dataset.

Table 2. Network architecture of the 2D-CNN model for air-writing recognition based on smart-band dataset.

\begin{tabular}{|c|c|c|c|c|c|c|c|c|}
\hline $\begin{array}{l}\text { Operation } \\
\text { Group }\end{array}$ & Layer Name & Filter Size & No. of Filters & Stride Size & Padding Size & $\begin{array}{l}\text { Activation } \\
\text { Function }\end{array}$ & Output Size * & $\begin{array}{c}\text { No. of } \\
\text { Parameters * }\end{array}$ \\
\hline- & Input & - & - & - & - & - & $200 \times 6 \times 1$ & 0 \\
\hline \multirow{4}{*}{ Group1 } & Conv1-1 & $2 \times 2$ & 32 & $1 \times 1$ & $1 \times 1$ & ReLU & $200 \times 6 \times 32$ & 160 \\
\hline & Conv1-2 & $2 \times 2$ & 32 & $1 \times 1$ & $1 \times 1$ & ReLU & $200 \times 6 \times 32$ & 4128 \\
\hline & MaxPool1 & $2 \times 2$ & 1 & $2 \times 2$ & 0 & - & $100 \times 3 \times 32$ & 0 \\
\hline & Dropout & & & $p=10 \%$ & & & $100 \times 3 \times 32$ & 0 \\
\hline \multirow{4}{*}{ Group2 } & Conv2-1 & $2 \times 2$ & 64 & $1 \times 1$ & $1 \times 1$ & ReLU & $100 \times 3 \times 64$ & 8256 \\
\hline & Conv2-2 & $2 \times 2$ & 64 & $1 \times 1$ & $1 \times 1$ & ReLU & $100 \times 3 \times 64$ & 16,448 \\
\hline & MaxPool2 & $2 \times 2$ & 1 & $2 \times 2$ & 0 & - & $50 \times 2 \times 64$ & 0 \\
\hline & Dropout & & & $p=20 \%$ & & & $50 \times 2 \times 64$ & 0 \\
\hline \multirow{4}{*}{ Group3 } & Conv3-1 & $2 \times 2$ & 128 & $1 \times 1$ & $1 \times 1$ & ReLU & $50 \times 2 \times 128$ & 32,896 \\
\hline & Conv3-2 & $2 \times 2$ & 128 & $1 \times 1$ & $1 \times 1$ & ReLU & $50 \times 2 \times 128$ & 65,664 \\
\hline & MaxPool3 & $2 \times 2$ & 1 & $2 \times 2$ & 0 & - & $25 \times 1 \times 128$ & 0 \\
\hline & Dropout & & & $p=20 \%$ & & & $25 \times 1 \times 128$ & 0 \\
\hline \multirow{4}{*}{ Group4 } & Flatten & - & - & - & - & - & 3200 & 0 \\
\hline & Dense & - & - & - & - & ReLU & 512 & $1,638,912$ \\
\hline & Dropout & & & $p=50 \%$ & & & 512 & 0 \\
\hline & Dense & - & - & - & - & Softmax & 26 & 13,338 \\
\hline
\end{tabular}

* Output size and no. of parameters vary based on the number of features and signal length, $l$, depending upon the dataset under consideration. For smart-band dataset, the number of features is 6 and the signal length, $l$ is 200 (see Table 1 and Section 3.2.1). Therefore, we yield this 2D-CNN network. The layers that construct the network and the attributes remain the same for all datasets.

\subsection{Experimental Settings and Evaluation Metrics}

To accelerate our training procedure, NVIDIA Tesla T4 GPU and 12 GB of RAM were used provided by Google Colaboratory free of charge [58]. We used OpenCV library [59] to interpolate the time-series data and Keras API over TensorFlow backend [60] to create the CNN model.

We measured the performance of the model by recognition accuracy (see Equation (7)) in user-dependent and user-independent principles. In the user-dependent principle, 10-fold cross-validation accuracy was reported for smart-band, RTC, and RTD datasets, and 5-fold cross-validation accuracy was reported for the variations of the 6DMG dataset 
to compare our method with the state-of-the-art methods. The evaluation metric was considered for multiclass classification, as we had a various number of digits and characters in different datasets (see Table 1). Note that the datasets are mostly balanced regarding the number of samples per letter per subject where applicable. Therefore, the evaluation of classification performance using accuracy alone was justified.

$$
\text { Accuracy }=\frac{\text { Number of correct predictions }}{\text { Total number of predictions }}
$$

\section{Experimental Analysis}

In this section, we experimented extensively with the various interpolation techniques to find the best method among them and a suitable fixed signal length, $l$ for interpolation using the smart-band dataset developed by Yanay et al. [2]. We performed comparative analysis with other existing signal length unification methods, such as padding and truncation, with the best interpolation method. Finally, we employed our methodology on six other air-writing datasets $[5,35,36]$ to verify our findings and yielded state-of-the-art performances.

\subsection{Searching Optimal Signal Length}

The process of recording the air-writing data results in different signal lengths. In Figure 2, we show a histogram of the lengths of all the samples from the smart-band dataset. Along with the interpolation methods, the fixed length of the signals plays an important role in data preprocessing, as we required a unified length to fit data into the 2D-CNN model.

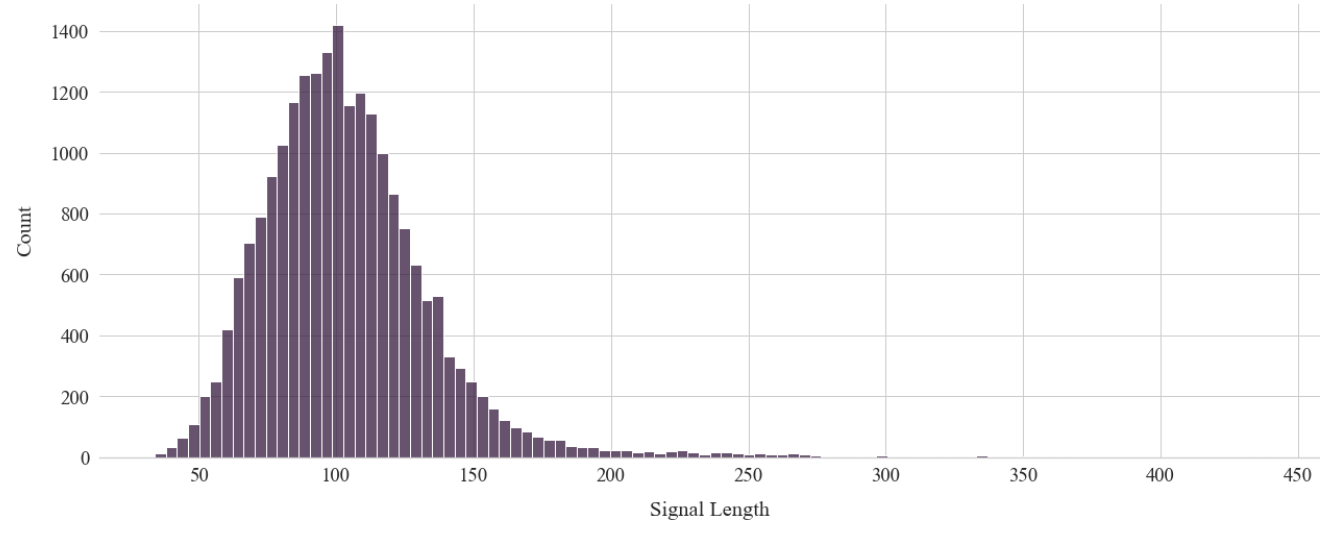

Figure 2. Histogram of the length of all samples from smart-band dataset.

Denoting the fixed signal length using $l$, we would have a matrix of size $l \times 6$ for each sample of the letters as we had six time-series data for each sample (see Section 3.1.1). Now, from the length distribution of each sample (see Figure 2), we can see that it is itself a matter of choice to select the fixed signal length (see Section 3.2.1). From the statistical characteristics, we considered the two following aspects. Firstly, we considered a round mean of the length distribution where approximately half of the data would be downsampled and half would be upsampled. However, from Section 3.2.2 and Figure 1, we can see that each interpolation method densely populates the time-series while upsampling, but for downsampling, we may significantly lose important data features. Therefore, secondly, if we upsample the majority of the data, we may have some data redundancy, but data loss is mostly prevented. Therefore, we considered 100 and 200 to be the signal length in our experimentation, where 100 is used to balance interpolation for upsampling and downsampling and 200 for upsampling the majority of data so that the data loss is minimized. Hence, the shape of the matrix containing all of the samples will be $21,450 \times 1 \times 6$, where $l=100,200$. 


\subsection{Effects of Various Interpolation Techniques}

For the signal length, $l=100$, we considered different methods for upsampling and downsampling as approximately half of the data was upsampled and half of the data was downsampled. Therefore, we had 20 different combinations of interpolation methods (see Table 3), whereas, for length $l=200$, considering 20 different combinations did not make any sense, as there are very little data left to be downsampled. So, we considered the same interpolation method for both upsampling and downsampling. To minimize the time taken to fine-tune the model, five random users (user no. $=\{22,10,4,47,40\}$ ) were taken to build the test set and the rest of the users remained in the training set. For different interpolation methods and fixed signal lengths, the performance of the classification model is presented in Table 3.

Table 3. Interpolation methods in different upsampling and downsampling settings.

\begin{tabular}{|c|c|c|c|c|}
\hline \multirow{2}{*}{$\begin{array}{l}\text { Upsampling } \\
\text { Method }\end{array}$} & \multirow{2}{*}{$\begin{array}{l}\text { Downsampling } \\
\text { Method }\end{array}$} & \multirow{2}{*}{ Signal Length, $l$} & \multicolumn{2}{|c|}{ Accuracy } \\
\hline & & & Avg. $(\%)$ & Std. \\
\hline \multirow{4}{*}{ Bicubic } & Bicubic & \multirow{4}{*}{100} & 87.35 & 0.41 \\
\hline & Lanczos & & 87.21 & 0.59 \\
\hline & Bilinear & & 87.76 & 0.45 \\
\hline & Nearest neighbor & & 87.04 & 0.21 \\
\hline \multirow{4}{*}{ Lanczos } & Bicubic & \multirow{4}{*}{100} & 87.54 & 0.18 \\
\hline & Lanczos & & 86.50 & 0.70 \\
\hline & Bilinear & & 86.84 & 0.46 \\
\hline & Nearest neighbor & & 86.73 & 0.27 \\
\hline \multirow{4}{*}{ Bilinear } & Bicubic & \multirow{4}{*}{100} & 86.91 & 0.77 \\
\hline & Lanczos & & 86.77 & 0.63 \\
\hline & Bilinear & & 86.25 & 0.80 \\
\hline & Nearest neighbor & & 87.38 & 0.09 \\
\hline \multirow{4}{*}{ Nearest neighbor } & Bicubic & \multirow{4}{*}{100} & 87.38 & 0.37 \\
\hline & Lanczos & & 87.32 & 0.76 \\
\hline & Bilinear & & 86.67 & 0.58 \\
\hline & Nearest neighbor & & 87.08 & 1.24 \\
\hline Bicubic & Bicubic & \multirow{4}{*}{200} & 88.54 & 0.31 \\
\hline Lanczos & Lanczos & & 87.35 & 0.31 \\
\hline Bilinear & Bilinear & & 88.46 & 0.19 \\
\hline Nearest neighbor & Nearest neighbor & & 88.08 & 0.59 \\
\hline
\end{tabular}

From Table 3, we can see that, using signal length, $l=100$, for all combinations of the interpolation methods, the results are marginally poor as we lose important temporal features for downsampling time-series data. For signal length, $l=200$, Bicubic interpolation for both the up and downsampling performs best, though the method did not yield data much compared to the other methods. As we upsampled most of the data for $l=200$, temporal data loss was minimal. Therefore, we had to select the optimal fixed signal length so that most of the data were upsampled. Consideration of the computation trade-off is critical as the signal length should not be too large to result in data redundancy.

In a similar setting, we performed comparative analysis with the other existing signal length unification methods such as pre-sequence padding and truncation and postsequence padding and truncation [7]. For a set of variable length signals, finding a unified length for all the signals may result in both padding (if the length of a particular signal is less than the fixed signal length) and truncation (if the length of a particular signal is greater than the fixed signal length). Here, the idea of padding and truncation is kind of similar to upsampling and downsampling, respectively. The sampling rate of the signal is changed accordingly for up and downsampling and the overall characteristics of the signal are kept where padding fills the extra slots by zeros and truncation cuts the signal to 
make the signal as required. The words, pre and post of the methods refer to the part of the sequence where the padding by zero or the truncation takes place.

In Table 4, it is shown that for a wide range of sequence lengths, the padding and truncation methods performed worse than the Bicubic interpolation technique. As the signal length increased to 400 , the inference time of the model and the number of floatingpoint operations also increased, but the performance of the model decreased for both cases of the padding and truncation method. Meanwhile, the Bicubic interpolation techniques performed much better for $l=200$, which is also computationally optimal. We also evaluated Bicubic interpolation for $l=50$ and $l=400$, and the method performed surprisingly well for $l=50$. In this case, pre-sequence padding and truncation and post-sequence padding and truncation were $59.87 \%$ and $48.15 \%$ accurate, respectively, where Bicubic interpolation achieved $84.94 \%$ accuracy. For $l=400$, the accuracy of the model was reduced than that of $l=200$ due to data redundancy. Therefore, we selected $l=200$ as the optimal signal length for the smart-band dataset. Following the same procedure, we also selected the signal length, $l$ for other datasets (see Table 5 ).

Table 4. Comparative analysis of Bicubic interpolation with padding and truncation methods.

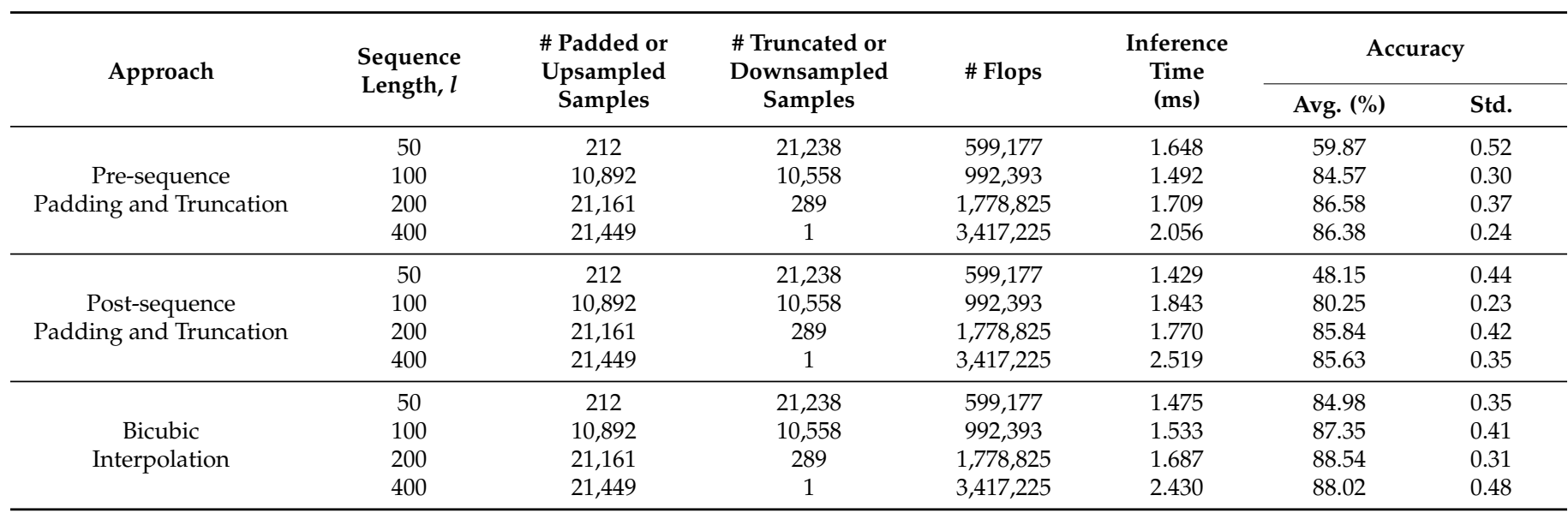

Table 5. Selected signal length, $l$ for all datasets.

\begin{tabular}{cccc}
\hline Dataset & Min & Max & Signal Length, $\boldsymbol{l}$ \\
\hline RTD & 18 & 150 & 125 \\
RTC & 21 & 173 & 125 \\
Smart-band & 34 & 438 & 200 \\
6DMG-digit & 29 & 218 & 175 \\
6DMG-lower & 27 & 163 & 150 \\
6DMG-upper & 27 & 412 & 250 \\
6DMG-all & 27 & 412 & 250
\end{tabular}

The terms "Min" and "Max" represent the minimum and maximum length of the signals in that particular dataset, respectively.

\subsection{Results and Discussions}

In the literature, air-writing recognition is evaluated under two different training principles $[2,16,33]$ : (1) the user-dependent principle and (2) the user-independent principle. The definitions of the principles are stated in the dataset description section (see Section 3.1).

Our method outperformed all the state-of-the-art methods in both principles for all the datasets. We reported the performances of our method in comparison with the previous methods [2,4,5,16,31,33,36,61-63], shown in Tables 6 and 7. From Table 6, compared with the existing methods employed on the smart-band, RTC, and RTD datasets, our proposed method achieved the best recognition performance in terms of accuracy in both userdependent and independent principles by a clear margin. For the variations of the 6DMG dataset, the performance comparison is presented in Table 7. Our method outperformed 
all the existing methods for digits, lowercase letters, uppercase letters, and all of these combined for both training principles. Empirically, under the user-dependent principle, our model achieved $100 \%$ accuracy for digits, which was verified by multiple random splits in cross-validation. Furthermore, we achieved a 3.55\% accuracy gain for all the classes combined and $0.52 \%, 1.62 \%$, and $2.24 \%$ accuracy gain for digit, lowercase, and uppercase datasets, respectively, in the user-independent principle.

Table 6. Performance evaluation for user-dependent and independent method on smart-band and RealSense-based Trajectory datasets. Abbreviations of the approaches are given in the Abbreviations section of this paper.

\begin{tabular}{ccccc}
\hline \multirow{2}{*}{ Training Principle } & Approach & \multicolumn{3}{c}{ Accuracy } \\
\cline { 3 - 5 } & & Smart-Band & RTC & RTD \\
\hline KNN-DTW based [2] & 89.20 & - & - \\
User-dependent & 2D-CNN based [36] & - & 97.29 & - \\
principle & LSTM based [5] & - & - & 99.17 \\
(10-fold CV) & CNN-LSTM fusion [31] & - & 98.74 & 99.63 \\
& Proposed & 91.34 & 99.63 & 99.76 \\
\hline User-independent & 1D-CNN [2] & 83.20 & - & - \\
principle & Proposed & 85.59 & - & - \\
\hline
\end{tabular}

Table 7. Performance evaluation for user-dependent and independent methods on the 6DMG dataset. Abbreviations of the approaches are given in the Abbreviations section of this paper.

\begin{tabular}{|c|c|c|c|c|c|c|c|c|c|}
\hline \multirow{3}{*}{$\begin{array}{l}\text { Training } \\
\text { Principle }\end{array}$} & \multirow{3}{*}{ Approach } & \multicolumn{8}{|c|}{ Accuracy } \\
\hline & & \multicolumn{2}{|c|}{ Digit } & \multicolumn{2}{|c|}{ Lower } & \multicolumn{2}{|c|}{ Upper } & \multicolumn{2}{|c|}{ All } \\
\hline & & Avg. (\%) & Std. & Avg. (\%) & Std. & Avg. (\%) & Std. & Avg. (\%) & Std. \\
\hline \multirow{7}{*}{$\begin{array}{c}\text { User- } \\
\text { dependent } \\
\text { principle } \\
(5 \text {-fold CV) }\end{array}$} & HMM-based [4] & - & - & - & - & 98.16 & 2.37 & - & - \\
\hline & LSTM-bases [61] & 97.33 & 1.49 & 96.80 & 0.57 & 98.34 & 0.50 & 94.75 & 0.31 \\
\hline & CRF-CNN fusion [62] & - & - & - & - & 98.57 & - & - & - \\
\hline & BiLSTM-CNN fusion [63] & 99.33 & - & - & - & 99.27 & - & - & - \\
\hline & CHMM-based [16] & 99.00 & 1.09 & 98.22 & 0.73 & 97.29 & 0.66 & 95.91 & 0.47 \\
\hline & UDA [33] & 99.78 & 0.03 & 98.94 & 0.08 & 99.55 & 0.06 & 97.03 & 0.11 \\
\hline & Proposed & 100.00 & 0.00 & 99.47 & 0.39 & 99.80 & 0.20 & 98.99 & 0.23 \\
\hline User- & CHMM [16] & 96.70 & 4.08 & 76.38 & 5.25 & 91.03 & 1.54 & 62.69 & 2.91 \\
\hline independent & UDA [33] & 98.74 & 0.34 & 92.86 & 0.48 & 96.99 & 0.45 & 87.69 & 0.58 \\
\hline principle & Proposed & 99.26 & 0.12 & 94.48 & 0.45 & 99.23 & 0.94 & 91.24 & 0.86 \\
\hline
\end{tabular}

\section{Conclusions}

Air-writing recognition will be essential in the post fourth industrial revolution world. In this study, we developed a method to recognize characters and digits in the English alphabet using time-series data. Sensor data preparation for deep learning methods while ensuring minimal data loss is a challenging task. We extensively explored different interpolation techniques which are widely used for images but often overlooked for time-series signals. Our experiment shows that interpolating the raw data using the Bicubic interpolation algorithm provides the best results in our use case. Upon this interpolated data, we trained our proposed 2D-CNN model to classify the letters, which outperformed the state-of-the-art methods by a clear margin. Fine tuning to the recognition system will be necessary before the real-world deployment of the air-writing recognition system. Furthermore, we can hybridize user-independent and user-dependent methods, create a guidance or feedback loop to the user, and introduce an auto-correction mechanism. Additionally, we can explore the subjects' characteristics extensively. Though the datasets particularly indicated some of the characteristics of the subjects, we did not consider those facts, as we intend to make the system more generalized. Last but not least, further research is essential to recognize words in similar settings, as it is much more challenging than recognition of an isolated character or digit. To build a fully functional air-writing recognition system, 
all of these issues have to be addressed. We present our work as one of the steppingstones in that path.

Author Contributions: Conceptualization, M.A.M.H. and F.A.A.; methodology, F.A.A. and M.A.M.H.; software, F.A.A. and M.A.S.; validation, F.A.A. and M.A.S.; formal analysis, F.A.A. and M.A.M.H.; investigation, F.A.A. and M.A.S.; resources, J.S. and A.S.; data curation and collection, M.A.M.H. and F.A.A.; writing—original draft preparation, F.A.A. and M.A.S.; writing—review and editing, F.A.A., M.A.M.H. and J.S.; visualization, F.A.A. and A.S.; supervision, A.S., M.A.M.H. and J.S.; project administration, J.S. and M.A.M.H.; funding acquisition, J.S. All authors have read and agreed to the published version of the manuscript.

Funding: This research received no external funding.

Institutional Review Board Statement: Not applicable.

Informed Consent Statement: Not applicable.

Data Availability Statement: All the datasets used in this research are publicly available. Smart-band dataset is available at: http://bigdatalab.tau.ac.il/shared_resources/datasets/airwriting_dataset.zip, accessed on 15 November 2021. All the variations of 6DMG dataset can be accessed at https: // mingyu623.github.io/6DMG.html, accessed on 15 November 2021, RTC dataset can be found at https://shahinur.com/en/rtc/, accessed on 15 November 2021, and RTD dataset is available at https://shahinur.com/en/rtd/, accessed on 15 November 2021.

Conflicts of Interest: The authors declare no conflict of interest.

\begin{tabular}{|c|c|}
\hline \\
\hline \multicolumn{2}{|c|}{ Abbreviations } \\
\hline RTD & RealSense Trajectory Digit \\
\hline RTC & RealSense Trajectory Character \\
\hline 6DMG & 6-Dimentional Motion Gesture \\
\hline $\mathrm{CNN}$ & Convolutional Neural Network \\
\hline KNN & K-Nearest Neighbors \\
\hline DTW & Dynamic-Time-Warping \\
\hline UDA & Unsupervised Domain Adaptation \\
\hline CRF & Conditional Random Fields \\
\hline HMM & Hidden Markov Model \\
\hline CHMM & Continuous Hidden Markov Model \\
\hline LSTM & Long Short-term Memory \\
\hline BiLSTM & Bidirectional Long Short-term Memory \\
\hline
\end{tabular}

\section{References}

1. Amma, C.; Schultz, T. Airwriting: Bringing text entry to wearable computers. XRDS 2013, 20, 50-55. [CrossRef]

2. Yanay, T.; Shmueli, E. Air-writing recognition using smart-bands. Pervasive Mob. Comput. 2020, 66, 101183. [CrossRef]

3. Zabulis, X.; Baltzakis, H.; Argyros, A.A. Vision-Based Hand Gesture Recognition for Human-Computer Interaction. Univers. Access Handb. 2009, 34, 30.

4. Chen, M.; AlRegib, G.; Juang, B.H. Air-Writing Recognition—Part I: Modeling and Recognition of Characters, Words, and Connecting Motions. IEEE Trans. Hum. Mach. Syst. 2016, 46, 403-413. [CrossRef]

5. Alam, M.; Kwon, K.C.; Abbass, M.Y.; Imtiaz, S.M.; Kim, N. Trajectory-based air-writing recognition using deep neural network and depth sensor. Sensors 2020, 20, 376. [CrossRef]

6. Liu, J.; Zhong, L.; Wickramasuriya, J.; Vasudevan, V. uWave: Accelerometer-based personalized gesture recognition and its applications. Pervasive Mob. Comput. 2009, 5, 657-675. [CrossRef]

7. Dwarampudi, M.; Reddy, N. Effects of padding on LSTMs and CNNs. arXiv 2019, arXiv:1903.07288.

8. Davis, P.J. Interpolation and Approximation; Courier Corporation: Chelmsford, MA, USA, 1975.

9. Aly, H.A.; Dubois, E. Image up-sampling using total-variation regularization with a new observation model. IEEE Trans. Image Process. 2005, 14, 1647-1659. [CrossRef]

10. Roy, R.; Pal, M.; Gulati, T. Zooming digital images using interpolation techniques. Int. J. Appl. Innov. Eng. Manag. 2013, 2, $34-45$.

11. Kim, J.; He, J.; Lyons, K.; Starner, T. The gesture watch: A wireless contact-free gesture based wrist interface. In Proceedings of the 2007 11th IEEE International Symposium on Wearable Computers, Boston, MA, USA, 11-13 October 2007; pp. 15-22. 
12. Ye, R.; Dai, Q. Implementing transfer learning across different datasets for time series forecasting. Pattern Recognit. 2021, 109, 107617. [CrossRef]

13. Luo, Y.; Liu, J.; Shimamoto, S. Wearable Air-Writing Recognition System employing Dynamic Time Warping. In Proceedings of the 2021 IEEE 18th Annual Consumer Communications \& Networking Conference (CCNC), Las Vegas, NV, USA, 9-12 January 2021; pp. 1-6.

14. MerlinLivingston, L.; Deepika, P.; Benisha, M. An Inertial Pen with Dynamic Time Warping Recognizer for Handwriting and Gesture Recognition. Int. J. Eng. Trends Technol. 2016, 35, 154-163.

15. Chen, M.; AlRegib, G.; Juang, B.H. Air-Writing Recognition-Part II: Detection and Recognition of Writing Activity in Continuous Stream of Motion Data. IEEE Trans. Hum. Mach. Syst. 2016, 46, 436-444. [CrossRef]

16. Xu, S.; Xue, Y. Air-writing characters modelling and recognition on modified CHMM. In Proceedings of the 2016 IEEE International Conference on Systems, Man, and Cybernetics (SMC), Budapest, Hungary, 9-12 October 2016; pp. 001510-001513.

17. Li, C.; Xie, C.; Zhang, B.; Chen, C.; Han, J. Deep Fisher discriminant learning for mobile hand gesture recognition. Pattern Recognit. 2018, 77, 276-288. [CrossRef]

18. Wen, H.; Ramos Rojas, J.; Dey, A.K. Serendipity: Finger gesture recognition using an off-the-shelf smartwatch. In Proceedings of the 2016 CHI Conference on Human Factors in Computing Systems, San Jose, CA, USA, 7-12 May 2016; pp. $3847-3851$.

19. Levy, A.; Nassi, B.; Elovici, Y.; Shmueli, E. Handwritten signature verification using wrist-worn devices. Proc. ACM Interact. Mob. Wearable Ubiquitous Technol. 2018, 2, 1-26. [CrossRef]

20. Amma, C.; Georgi, M.; Schultz, T. Airwriting: A wearable handwriting recognition system. Pers. Ubiquitous Comput. 2014, 18, 191-203. [CrossRef]

21. Xu, C.; Pathak, P.H.; Mohapatra, P. Finger-writing with smartwatch: A case for finger and hand gesture recognition using smartwatch. In Proceedings of the 16th International Workshop on Mobile Computing Systems and Applications, Santa Fe, NM, USA, 12-13 February 2015; pp. 9-14.

22. Lin, X.; Chen, Y.; Chang, X.W.; Liu, X.; Wang, X. Show: Smart handwriting on watches. Proc. ACM Interact. Mob. Wearable Ubiquitous Technol. 2018, 1, 1-23. [CrossRef]

23. Chen, Y.H.; Huang, C.H.; Syu, S.W.; Kuo, T.Y.; Su, P.C. Egocentric-View Fingertip Detection for Air Writing Based on Convolutional Neural Networks. Sensors 2021, 21, 4382. [CrossRef] [PubMed]

24. Kim, U.H.; Hwang, Y.; Lee, S.K.; Kim, J.H. Writing in The Air: Unconstrained Text Recognition from Finger Movement Using Spatio-Temporal Convolution. arXiv 2021, arXiv:2104.09021.

25. Bastas, G.; Kritsis, K.; Katsouros, V. Air-Writing Recognition using Deep Convolutional and Recurrent Neural Network Architectures. In Proceedings of the 2020 17th International Conference on Frontiers in Handwriting Recognition (ICFHR), Dortmund, Germany, 8-10 September 2020; pp. 7-12.

26. Tsai, T.H.; Hsieh, J.W.; Chang, C.W.; Lay, C.R.; Fan, K.C. Air-writing recognition using reverse time ordered stroke context. J. Vis. Commun. Image Represent. 2021, 78, 103065. [CrossRef]

27. Arsalan, M.; Santra, A.; Bierzynski, K.; Issakov, V. Air-Writing with Sparse Network of Radars using Spatio-Temporal Learning. In Proceedings of the 2020 25th International Conference on Pattern Recognition (ICPR), Milan, Italy, 10-15 January 2021; pp. 8877-8884.

28. Moazen, D.; Sajjadi, S.A.; Nahapetian, A. AirDraw: Leveraging smart watch motion sensors for mobile human computer interactions. In Proceedings of the 2016 13th IEEE Annual Consumer Communications \& Networking Conference (CCNC), Las Vegas, NV, USA, 9-12 January 2016; pp. 442-446.

29. Uysal, C.; Filik, T. RF-Wri: An Efficient Framework for RF-Based Device-Free Air-Writing Recognition. IEEE Sens. J. 2021, 21, 17906-17916. [CrossRef]

30. Hsieh, C.H.; Lo, Y.S.; Chen, J.Y.; Tang, S.K. Air-Writing Recognition Based on Deep Convolutional Neural Networks. IEEE Access 2021, 9, 142827-142836. [CrossRef]

31. Alam, M.S.; Kwon, K.C.; Imtiaz, S.M.; Hossain, M.B.; Rupali, S.; Hyun, J. Air-writing recognition using a fusion CNN-LSTM neural network. In Proceedings of the 8th International Conference on Electronics, Electrical Engieering, Computer Scicence: Innovationa and Convergence, Osaka, Japan, 21-23 July 2021.

32. Alam, M.S.; Kwon, K.C.; Kim, N. Implementation of a Character Recognition System Based on Finger-Joint Tracking Using a Depth Camera. IEEE Trans. Hum. Mach. Syst. 2021, 51, 229-241. [CrossRef]

33. Xu, S.; Xue, Y.; Zhang, X.; Jin, L. A Novel Unsupervised domain adaptation method for inertia-Trajectory translation of in-air handwriting. Pattern Recognit. 2021, 116, 107939. [CrossRef]

34. Lepot, M.; Aubin, J.B.; Clemens, F.H. Interpolation in time series: An introductive overview of existing methods, their performance criteria and uncertainty assessment. Water 2017, 9, 796. [CrossRef]

35. Chen, M.; AlRegib, G.; Juang, B.H. 6dmg: A new 6d motion gesture database. In Proceedings of the 3rd Multimedia Systems Conference, Chapel Hill, NC, USA, 22-24 February 2012; pp. 83-88.

36. Alam, M.S.; Kwon, K.; Kim, N. Trajectory-Based Air-Writing Character Recognition Using Convolutional Neural Network. In Proceedings of the 4th International Conference on Control, Robotics and Cybernetics, CRC 2019, Tokyo, Japan, 27-30 September 2019; pp. 86-90. [CrossRef]

37. Simonyan, K.; Zisserman, A. Very deep convolutional networks for large-scale image recognition. arXiv 2014, arXiv:1409.1556.

38. Wu, H.; Gu, X. Towards Dropout Training for Convolutional Neural Networks. Neural Netw. 2015, 71, 1-10. [CrossRef] [PubMed] 
39. Park, S.; Kwak, N. Analysis on the dropout effect in convolutional neural networks. In Asian Conference on Computer Vision; Springer: Berlin/Heidelberg, Germany, 2016; pp. 189-204.

40. Keys, R. Cubic convolution interpolation for digital image processing. IEEE Trans. Acoust Speech Signal Process. 1981, 29, 1153-1160. [CrossRef]

41. Burger, W.; Burge, M.J. Digital Image Processing: An Algorithmic Introduction Using Java; Springer: Berlin/Heidelberg, Germany, 2016.

42. Athawale, T.; Entezari, A. Uncertainty quantification in linear interpolation for isosurface extraction. IEEE Trans. Vis. Comput. Graph. 2013, 19, 2723-2732. [CrossRef]

43. Carrizosa, E.; Olivares-Nadal, A.V.; Ramírez-Cobo, P. Time series interpolation via global optimization of moments fitting. Eur. J. Oper. Res. 2013, 230, 97-112. [CrossRef]

44. Gnauck, A. Interpolation and approximation of water quality time series and process identification. Anal. Bioanal. Chem. 2004, 380, 484-492. [CrossRef]

45. Hubel, D.H.; Wiesel, T.N. Receptive fields and functional architecture of monkey striate cortex. J. Physiol. 1968, 195, 215-243. [CrossRef]

46. Fukushima, K.; Miyake, S. Neocognitron: A self-organizing neural network model for a mechanism of visual pattern recognition. In Competition and Cooperation in Neural Nets; Springer: Berlin/Heidelberg, Germany, 1982; pp. 267-285.

47. Zeng, M.; Nguyen, L.T.; Yu, B.; Mengshoel, O.J.; Zhu, J.; Wu, P.; Zhang, J. Convolutional neural networks for human activity recognition using mobile sensors. In Proceedings of the 6th International Conference on Mobile Computing, Applications and Services, Austin, TX, USA, 6-7 November 2014; pp. 197-205.

48. Duffner, S.; Berlemont, S.; Lefebvre, G.; Garcia, C. 3D gesture classification with convolutional neural networks. In Proceedings of the 2014 IEEE International Conference on Acoustics, Speech and Signal Processing (ICASSP), Florence, Italy, 4-9 May 2014; pp. 5432-5436.

49. Yang, J.; Nguyen, M.N.; San, P.P.; Li, X.; Krishnaswamy, S. Deep Convolutional Neural Networks on Multichannel Time Series for Human Activity Recognition. In Proceedings of the Twenty-Fourth International Joint Conference on Artificial Intelligence, IJCAI 2015, Buenos Aires, Argentina, 25-31 July 2015; pp. 3995-4001.

50. Ha, S.; Choi, S. Convolutional neural networks for human activity recognition using multiple accelerometer and gyroscope sensors. In Proceedings of the 2016 International Joint Conference on Neural Networks (IJCNN), Vancouver, BC, Canada, 24-29 July 2016; pp. 381-388.

51. Lee, S.M.; Yoon, S.M.; Cho, H. Human activity recognition from accelerometer data using Convolutional Neural Network. In Proceedings of the 2017 IEEE International Conference on Big Data and Smart Computing, Jeju, Korea, 13-16 February 2017; pp. 131-134.

52. Panwar, M.; Dyuthi, S.R.; Prakash, K.C.; Biswas, D.; Acharyya, A.; Maharatna, K.; Gautam, A.; Naik, G.R. CNN based approach for activity recognition using a wrist-worn accelerometer. In Proceedings of the 2017 39th Annual International Conference of the IEEE Engineering in Medicine and Biology Society (EMBC), Jeju Island, Korea, 11-15 July 2017; pp. 2438-2441.

53. Li, T.; Zhang, Y.; Wang, T. SRPM-CNN: A combined model based on slide relative position matrix and CNN for time series classification. Complex Intell. Syst. 2021, 7, 1619-1631. [CrossRef]

54. Krizhevsky, A.; Sutskever, I.; Hinton, G.E. Imagenet classification with deep convolutional neural networks. Adv. Neural Inf. Process. Syst. 2012, 25, 1097-1105. [CrossRef]

55. Howard, A.G.; Zhu, M.; Chen, B.; Kalenichenko, D.; Wang, W.; Weyand, T.; Andreetto, M.; Adam, H. Mobilenets: Efficient convolutional neural networks for mobile vision applications. arXiv 2017, arXiv:1704.04861.

56. Szegedy, C.; Liu, W.; Jia, Y.; Sermanet, P.; Reed, S.; Anguelov, D.; Erhan, D.; Vanhoucke, V.; Rabinovich, A. Going deeper with convolutions. In Proceedings of the IEEE Conference on Computer Vision and Pattern Recognition, Boston, MA, USA, 7-12 June 2015; pp. 1-9.

57. Srivastava, N.; Hinton, G.; Krizhevsky, A.; Sutskever, I.; Salakhutdinov, R. Dropout: A simple way to prevent neural networks from overfitting. J. Mach. Learn. Res. 2014, 15, 1929-1958.

58. Carneiro, T.; Medeiros Da NóBrega, R.V.; Nepomuceno, T.; Bian, G.B.; De Albuquerque, V.H.C.; Filho, P.P.R. Performance Analysis of Google Colaboratory as a Tool for Accelerating Deep Learning Applications. IEEE Access 2018, 6, 61677-61685. [CrossRef]

59. Bradski, G. The OpenCV Library. J. Softw. Tools 2000. Available online: https://github.com/opencv/opencv (accessed on 21 November 2021).

60. Chollet, F. Keras. 2015. Available online: https://github.com/fchollet/keras (accessed on 21 November 2021).

61. Xu, S.; Xue, Y. A Long Term Memory Recognition Framework on Multi-Complexity Motion Gestures. In Proceedings of the 14th IAPR International Conference on Document Analysis and Recognition, ICDAR 2017, Kyoto, Japan, 9-15 November 2017; pp. 201-205. [CrossRef]

62. Yang, C.; Ku, B.; Han, D.; Ko, H. Alpha-numeric hand gesture recognition based on fusion of spatial feature modelling and temporal feature modelling. Electron. Lett. 2016, 52, 1679-1681. [CrossRef]

63. Yana, B.; Onoye, T. Fusion networks for air-writing recognition. In International Conference on Multimedia Modeling; Springer: Berlin/Heidelberg, Germany, 2018; pp. 142-152. 